\title{
Analysis of a predator-prey model with impulsive diffusion and releasing on predator population
}

\author{
Airen Zhou ${ }^{1,2}$, Pairote Sattayatham ${ }^{1}$ and Jianjun Jiao ${ }^{2^{*}}$
}

\section{"Correspondence:}

jiaojianjun05@126.com

${ }^{2}$ Guizhou Key Laboratory of

Economic System Simulation,

School of Mathematics and

Statistics, Guizhou University of

Finance and Economics, Guiyang,

550004, P.R. China

Full list of author information is

available at the end of the article

\section{每 Springer}

\begin{abstract}
In this paper, we establish a predator-prey model with impulsive diffusion and releasing on predator population. This predator-prey model for two regions, which are connected by diffusion of predator population, portrays the evolvement of population. We prove that all solutions of the investigated system are uniformly ultimately bounded. We also prove that there exists globally asymptotically stable prey-extinction boundary periodic solution. The condition for permanence is obtained. Simulations are also employed to verify our results. It is discovered that the increasing diffusive rate of predator population will count against the pest management. We conclude that the impulsive diffusion and releasing predator provide reliable tactic basis for pest management.
\end{abstract}

Keywords: predator-prey model; impulsive diffusion; impulsive releasing; extinction; permanence

\section{Introduction}

The warfare between human and pests has sustained for thousands of years. In the past few decades, man have adopted some advanced and modern weapons for instance chemical pesticides, biological pesticides, remote sensing and measure, computers, atomic energy, et cetera. Some brilliant achievements have been obtained. However, the warfare will never be over. Although a great number of pesticides were used to control pests, the insect pests impairing crops are increasing for the resistance to pesticides. With pesticides employed, the residual pests breed a large number of pests with resistance to pesticides. So the pesticide is invalid in some sense. Moreover, insect pests will continue. On the other hand, the chemical pesticides kill not only pests but also their natural enemies. Therefore, insect pests are rampant again. Then the effect of chemical control was challenged. Furthermore, the practice proves that long-term adopting chemical control may give rise to disastrous results, for example, environmental contamination and toxicosis of the man and animals and so on.

The use of natural enemy to suppress pests is one of the most important approaches in pest control. Biological control [1-8] is one of the reduction in pest populations from the actions of other living organisms, often called natural enemies or beneficial species. It is the purposeful introduction and establishment of one or more natural enemies from

(c) 2016 Zhou et al. This article is distributed under the terms of the Creative Commons Attribution 4.0 International License (http://creativecommons.org/licenses/by/4.0/), which permits unrestricted use, distribution, and reproduction in any medium, provided you give appropriate credit to the original author(s) and the source, provide a link to the Creative Commons license, and indicate if changes were made. 
region of origin of an exotic pest, specifically for the purpose of suppressing the abundance of the pest in a new target region to a level at which it no longer causes economic damage. Jiao et al. [9] analyzed the dynamics of a stage-structured Holling mass defence predatorprey model with impulsive perturbations on predators

$$
\left\{\begin{array}{l}
x_{1}^{\prime}(t)=r x_{2}(t)-r e^{-w \tau_{1}} x_{2}\left(t-\tau_{1}\right)-w x_{1}(t), \\
x_{2}^{\prime}(t)=r e^{-w \tau_{1}} x_{2}\left(t-\tau_{1}\right)-\frac{\beta x_{2}(t)}{1+a x_{2}+b x_{2}^{2}} x_{3}(t)-d_{3} x_{2}(t)-d_{4} x_{2}^{2}(t), \\
x_{3}^{\prime}(t)=\frac{k \beta x_{2}(t)}{1+a x_{2}+b x_{2}^{2}} x_{3}(t)-d x_{3}(t), \\
\Delta x_{1}(t)=0, \\
\Delta x_{2}(t)=0, \\
\Delta x_{3}(t)=\mu, \\
\left(\varphi_{1}(\zeta), \varphi_{2}(\zeta), \varphi_{3}(\zeta)\right) \in C_{+}=C\left(\left[-\tau_{1}, 0\right], R_{+}^{3}\right), \quad \varphi_{i}(0)>0, i=1,2,3,
\end{array}\right.
$$

where $x_{1}(t)$ and $x_{2}(t)$ represent the immature and mature pest densities, respectively, and $x_{3}(t)$ denotes the density of nature enemy. The biological meanings of parameters can be seen in reference [9].

The dispersal is a ubiquitous phenomenon in the natural world. It is important for us to understand the ecological and evolutionary dynamics of populations mirrored by the large number of mathematical models devoted to it in the scientific literature [10-13]. In recent years, the analysis of these models focus on the coexistence of population and local (or global) stability of equilibria [14-20]. Spatial factors play a fundamental role in the persistence and stability of the population, although the complete results have not yet been obtained even in the simplest one-species case. Whereas the population dynamics with the effects of spatial heterogeneity is modeled by a diffusion process, most previous papers focused on the population dynamical system modeled by the ordinary differential equations. But in practice, it is often the case that diffusion occurs in regular pulse. For example, when winter comes, birds migrate between patches in search for a better environment,whereas they do not diffuse in other seasons, and the excursion of foliage seeds occurs at fixed period of time every year. Thus, impulsive diffusion provides a more natural description. Lately theories of impulsive differential equations [21] have been introduced into population dynamics. Jiao et al. [22] propose to investigate the dynamical behaviors of a stage-structured predator-prey model with prey impulsively diffusing between two patches

$$
\left\{\begin{array}{l}
\frac{d x_{1}(t)}{d t}=x_{1}(t)\left(a_{1}-b_{1} x_{1}(t)\right), \\
\frac{d x_{2}(t)}{d t}=x_{2}(t)\left(a_{2}-b_{2} x_{2}(t)\right)-\alpha x_{2}(t) y_{2}(t), \\
\frac{d y_{1}(t)}{d t}=k \alpha x_{2}(t) y_{2}(t)-k \alpha e^{-w \tau_{1}} x_{2}\left(t-\tau_{1}\right) y_{2}\left(t-\tau_{1}\right)-w y_{1}(t), \\
\frac{d y_{2}(t)}{d t}=k \alpha e^{-w \tau_{1}} x_{2}\left(t-\tau_{1}\right) y_{2}\left(t-\tau_{1}\right)-d y_{2}(t), \\
\Delta x_{1}(t)=d_{1}\left(x_{2}(t)-x_{1}(t)\right), \\
\Delta x_{2}(t)=d_{2}\left(x_{1}(t)-x_{2}(t)\right), \\
\Delta y_{1}(t)=0, \\
\Delta y_{2}(t)=0,
\end{array}\right\} \quad t=n \tau, n=1,2, \ldots,
$$

where we suppose that the system is composed of two patches connected by diffusion and occupied by a single species $x_{i}(i=1,2)$ is the density of species in the $i$ th patch, and $y_{1}(t)$ and $y_{2}(t)$ represent the densities of the immature individual predator and mature 
individual predator at time $t$ in the second patch. The biological meanings of parameters can be seen in reference [22].

Theories of impulsive differential equations have been introduced into population dynamics lately [23-28]. Impulsive equations are found in almost every domain of applied science and have been studied in many investigations [28-33]; they generally describe phenomena that are subject to steep or instantaneous changes. The theories of population dynamical systems and their applications have achieved many good results. In this paper, we investigate a predator-prey model with impulsive diffusion and releasing on predator population. We expect to obtain some dynamical properties of the investigated system. We also expect that the impulsive diffusion and releasing predator will provide reliable tactic basis for pest management.

The organization of this paper is as follows. In the next section, we introduce the model and background concepts. In Section 3, some important lemmas are presented. We give the globally asymptotically stable conditions of the prey-extinction boundary periodic solution of system (3) and the permanent condition of system (3) in Section 4. Simulation analysis and brief discussion are given in the last section to conclude this work.

\section{The model}

In this paper, we establish a predator-prey model with impulsive diffusion and releasing on predator population:

$$
\left\{\begin{array}{l}
\frac{d x_{1}(t)}{d t}=x_{1}(t)\left(a_{1}-b_{1} x_{1}(t)\right)-\frac{\beta_{1} x_{1}(t) y_{1}(t)}{\sigma_{1}+x_{1}(t)}, \\
\frac{d y_{1}(t)}{d t}=\frac{k_{1} \beta_{1} x_{1}(t) y_{1}(t)}{\sigma_{1}+x_{1}(t)}-d_{1} y_{1}(t), \\
\frac{d x_{2}(t)}{d t}=x_{2}(t)\left(a_{2}-b_{2} x_{2}(t)\right)-\frac{\beta_{2} x_{2}(t) y_{2}(t)}{\sigma_{2}+x_{2}(t)}, \\
\frac{d y_{2}(t)}{d t}=\frac{k_{2} \beta_{2} x_{2}(t) y_{2}(t)}{\sigma_{2}+x_{2}(t)}-d_{2} y_{2}(t), \\
\Delta x_{1}(t)=0 \\
\Delta y_{1}(t)=D\left(y_{2}(t)-y_{1}(t)\right), \\
\Delta x_{2}(t)=0 \\
\Delta y_{2}(t)=D\left(y_{1}(t)-y_{2}(t)\right), \\
\Delta x_{1}(t)=0, \\
\Delta y_{1}(t)=\mu_{1}, \\
\Delta x_{2}(t)=0, \quad t=(n+l) \tau, n \in Z_{+}, \\
\Delta y_{2}(t)=\mu_{2},
\end{array}\right\} \quad t=(n+1) \tau, n \in Z_{+},
$$

where we suppose that the system is composed of two patches connected by diffusion. These two patches are separated by rivers or highways or railways. The predator population can traverse the rivers or highways or railways, whereas the prey population not. In this system, $x_{i}(t)$ and $y_{i}(t)$ represent the numbers of prey and predator populations in patch $i(i=1,2)$ at time $t, a_{i}>0$ represents the intrinsic growth rate of the prey population in patch $i(i=1,2)$, and $b_{i}>0$ represents the coefficient of the intraspecific competition of the prey population in patch $i(i=1,2)$. The predator consumes the prey according to Holling type-II functional response

$$
\frac{\beta_{i} x_{i}(t)}{\sigma_{i}+x_{i}(t)} \quad(i=1,2)
$$


with the half-saturation constant $\sigma_{i}$ in patch $i(i=1,2)$ at time $t . k_{i}(i=1,2)$ is the rate of conversion of nutrients into the reproduction of the predator in patch $i(i=1,2), d_{i}(i=1,2)$ represents the death in patch $i(i=1,2)$. The pulse diffusion occurs every $\tau$ period ( $\tau$ is a positive constant), the system evolves from its initial state without being further affected by diffusion until the next pulse appears; $\Delta y_{i}((n+l) \tau)=y_{i}\left((n+l) \tau^{+}\right)-y_{i}((n+l) \tau)$, where $y_{i}\left((n+l) \tau^{+}\right)$represents the density of population in the $i$ th patch immediately after the $n$th diffusion pulse at time $t=(n+l) \tau$, whereas $y_{i}((n+l) \tau)$ represents the density of population in the $i$ th patch before the $n$th diffusion pulse at time $t=(n+l) \tau, 0<l<1, n \in Z_{+}, 0<D<1$ represents the diffusive rate between the patches, $\Delta y_{i}((n+1) \tau)=y_{i}\left((n+1) \tau^{+}\right)-y_{i}((n+1) \tau)$, and $\mu_{i}(i=1,2)$ represents the releasing amount of predator population at $t=(n+1) \tau$, $n \in Z_{+}$in patch $i(i=1,2)$.

\section{The lemmas}

The solution of (3), denoted by $X(t)=\left(x_{1}(t), y_{1}(t), x_{2}(t), y_{2}(t)\right)^{T}$, is a piecewise continuous function $X: R_{+} \rightarrow R_{+}^{4}, X(t)$ is continuous on $(n \tau,(n+l) \tau]$ and $((n+l) \tau,(n+1) \tau], n \in Z_{+}$, and $X\left(n \tau^{+}\right)=\lim _{t \rightarrow n \tau^{+}} X(t), X\left((n+l) \tau^{+}\right)=\lim _{t \rightarrow(n+l) \tau^{+}} X(t)$ exist. Obviously, the global existence and uniqueness of solutions of (3) is guaranteed by the smoothness properties of $f$, the mapping defined by the right side of system (3) [21].

Let $V: R_{+} \times R_{+}^{4} \rightarrow R_{+}$. Then $V$ is said to belong to class $V_{0}$ if

(i) $V$ is continuous in $(n \tau,(n+l) \tau] \times R_{+}^{4}$ and $((n+l) \tau,(n+1) \tau] \times R_{+}^{4}$ for all $z \in R_{+}^{4}$,

$n \in Z_{+}$, and $V\left(n \tau^{+}, z\right)=\lim _{(t, y) \rightarrow\left(n \tau^{+}, z\right)} V(t, y)$ and

$V\left((n+l) \tau^{+}, z\right)=\lim _{(t, y) \rightarrow\left((n+l) \tau^{+}, y\right)} V(t, y)$ exist;

(ii) $V$ is locally Lipschitzian in $z$.

Definition 3.1 If $V \in V_{0}$, then, for $(t, z) \in(n \tau,(n+l) \tau] \times R_{+}^{4}$ and $((n+l) \tau,(n+1) \tau] \times R_{+}^{4}$, the upper right derivative of $V(t, z)$ with respect to the impulsive differential system (3) is defined as

$$
D^{+} V(t, z)=\limsup _{h \rightarrow 0} \frac{1}{h}[V(t+h, z+h f(t, z))-V(t, z)] .
$$

Since $\frac{d x_{i}(t)}{d t}=0$ when $x_{i}(t)=0, \frac{d y_{i}(t)}{d t}=0$ when $y_{i}(t)=0$, and $\Delta y_{i}(t)=\mu_{i}>0$ when $t=$ $(n+1) \tau$, we easily obtain the following lemma.

Lemma 3.2 Suppose that $X(t)$ is a solution of (3) with $X\left(0^{+}\right) \geq 0$. Then $X(t) \geq 0$ for $t \geq 0$, and further $X(t)>0(t \geq 0)$ for $X\left(0^{+}\right)>0$.

Lemma 3.3 [21] Let the function $m \in P C^{\prime}\left[R^{+}, R\right]$ satisfy the inequalities

$$
\left\{\begin{array}{l}
m^{\prime}(t) \leq p(t) m(t)+q(t), \quad t \geq t_{0}, t \neq t_{k}, k=1,2, \ldots, \\
m\left(t_{k}^{+}\right) \leq d_{k} m\left(t_{k}\right)+b_{k}, \quad t=t_{k},
\end{array}\right.
$$

where $p, q \in C\left[R^{+}, R\right]$, and $d_{k} \geq 0$ and $b_{k}$ are constants. Then

$$
\begin{aligned}
m(t) \leq & m\left(t_{0}\right) \prod_{t_{0}<t_{k}<t} d_{k} \exp \left(\int_{t_{0}}^{t} p(s) d s\right)+\sum_{t_{0}<t_{k}<t}\left(\prod_{t_{k}<t_{j}<t} d_{j} \exp \left(\int_{t_{k}}^{t} p(s) d s\right)\right) b_{k} \\
& +\int_{t_{0}}^{t} \prod_{s<t_{k}<t} d_{k} \exp \left(\int_{s}^{t} p(\sigma) d \sigma\right) q(s) d s, \quad t \geq t_{0} .
\end{aligned}
$$


Now, we show that all solutions of (3) are uniformly ultimately bounded.

Lemma 3.4 There exists a constant $M>0$ such that $x_{i}(t) \leq M, y_{i}(t) \leq M(i=1,2)$ for each solution $\left(x_{1}(t), y_{1}(t), x_{2}(t), y_{2}(t)\right)$ of $(3)$ with all t large enough.

Proof Define

$V(t)=k_{1} x_{1}(t)+y_{1}(t)+k_{2} x_{2}(t)+y_{2}(t)$,

and $\lambda=\min _{i=1,2}\left\{d_{i}\right\}$. When $t \neq n \tau, t \neq(n+l) \tau$, we have

$$
\begin{aligned}
D^{+} V(t) & +\lambda V(t) \\
= & k_{1} x_{1}(t)\left[\left(a_{1}+\lambda\right)-b_{1} x_{1}(t)\right]-\left(d_{1}-\lambda\right) y_{1}(t) \\
& +k_{2} x_{2}(t)\left[\left(a_{2}+\lambda\right)-b_{2} x_{2}(t)\right]-\left(d_{2}-\lambda\right) y_{2}(t) \\
\leq & k_{1} x_{1}(t)\left[\left(a_{1}+\lambda\right)-b_{1} x_{1}(t)\right]+k_{2} x_{2}(t)\left[\left(a_{2}+\lambda\right)-b_{2} x_{2}(t)\right] \\
= & -k_{1} b_{1}\left(x_{1}(t)-\frac{a_{1}+\lambda}{2 b_{1}}\right)^{2}+\frac{k_{1}\left(a_{1}+\lambda\right)^{2}}{4 b_{1}} \\
& \quad-k_{2} b_{2}\left(x_{2}(t)-\frac{a_{2}+\lambda}{2 b_{2}}\right)^{2}+\frac{k_{2}\left(a_{2}+\lambda\right)^{2}}{4 b_{2}} \\
\leq & \frac{k_{1}\left(a_{1}+\lambda\right)^{2}}{4 b_{1}}+\frac{k_{2}\left(a_{2}+\lambda\right)^{2}}{4 b_{2}} \triangleq \zeta .
\end{aligned}
$$

When $t=n \tau$, we have

$$
\begin{aligned}
V\left(n \tau^{+}\right) & =k_{1} x_{1}\left(n \tau^{+}\right)+y_{1}\left(n \tau^{+}\right)+k_{2} x_{2}\left(n \tau^{+}\right)+y_{2}\left(n \tau^{+}\right) \\
& =k_{1} x_{1}(n \tau)+y_{1}(n \tau)+\mu_{1}+k_{2} x_{2}(n \tau)+y_{2}(n \tau)+\mu_{2} \\
& =k_{1} x_{1}(n \tau)+y_{1}(n \tau)+k_{2} x_{2}(n \tau)+y_{2}(n \tau)+\mu_{1}+\mu_{2} \\
& =V(n \tau)+\left(\mu_{1}+\mu_{2}\right) .
\end{aligned}
$$

When $t=(n+l) \tau$, we have

$$
\begin{aligned}
V\left((n+l) \tau^{+}\right)= & k_{1} x_{1}\left((n+l) \tau^{+}\right)+y_{1}\left((n+l) \tau^{+}\right)+k_{2} x_{2}\left((n+l) \tau^{+}\right)+y_{2}\left((n+l) \tau^{+}\right) \\
= & k_{1} x_{1}((n+l) \tau)+(1-D) y_{1}((n+l) \tau)+D y_{2}((n+l) \tau)+k_{2} x_{2}((n+l) \tau) \\
& +D y_{1}((n+l) \tau)+(1-D) y_{2}((n+l) \tau) \\
= & k_{1} x_{1}((n+l) \tau)+y_{1}((n+l) \tau)+k_{2} x_{2}((n+l) \tau)+y_{2}((n+l) \tau) \\
= & V((n+l) \tau) .
\end{aligned}
$$

By Lemma 3.3, for $t \in(n \tau,(n+1) \tau]$, we have

$$
\begin{aligned}
V(t) & \leq V\left(0^{+}\right) e^{-\lambda t}+\frac{\zeta}{\lambda}\left(1-e^{-\lambda t}\right)+\left(\mu_{1}+\mu_{2}\right) \frac{e^{-\lambda(t-\tau)}}{1-e^{\lambda \tau}}+\left(\mu_{1}+\mu_{2}\right) \frac{e^{\lambda \tau}}{e^{\lambda \tau}-1} \\
& \rightarrow \frac{\zeta}{\lambda}+\left(\mu_{1}+\mu_{2}\right) \frac{e^{\lambda \tau}}{e^{\lambda \tau}-1} \quad \text { as } t \rightarrow \infty .
\end{aligned}
$$


So $V(t)$ is uniformly ultimately bounded. Hence, by the definition of $V(t)$ we have that there exists a constant $M>0$ such that $x_{i}(t) \leq M, y_{i}(t) \leq M(i=1,2)$ for $t$ large enough. The proof is complete.

If $x_{i}(t)=0(i=1,2)$, then we have the subsystem of (3)

$$
\left\{\begin{array}{l}
\left.\begin{array}{l}
\frac{d y_{1}(t)}{d t}=-d_{1} y_{1}(t), \\
\frac{d y_{2}(t)}{d t}=-d_{2} y_{2}(t),
\end{array}\right\} \quad t \neq(n+l) \tau, t \neq(n+1) \tau \\
\Delta y_{1}(t)=D\left(y_{2}(t)-y_{1}(t)\right), \\
\Delta y_{2}(t)=D\left(y_{1}(t)-y_{2}(t)\right), \\
\left.\begin{array}{c}
\Delta y_{1}(t)=-\mu_{1}, \\
\Delta y_{2}(t)=-\mu_{2},
\end{array}\right\} \quad t=(n+1) \tau, n=1,2, \ldots
\end{array}\right.
$$

We easily obtain the analytic solution of (5) between pulses as follows:

$$
\left\{\begin{array}{l}
y_{1}(t)= \begin{cases}y_{1}\left(n \tau^{+}\right) e^{-d_{1}(t-n \tau)}, & t \in(n \tau,(n+l) \tau], \\
y_{1}\left((n+l) \tau^{+}\right) e^{-d_{1}(t-(n+l) \tau)}, & t \in((n+l) \tau,(n+1) \tau],\end{cases} \\
y_{2}(t)= \begin{cases}y_{2}\left(n \tau^{+}\right) e^{-d_{2}(t-n \tau)}, \\
y_{2}\left((n+l) \tau^{+}\right) e^{-d_{2}(t-(n+l) \tau)}, & t \in((n+l) \tau,(n+1) \tau] .\end{cases}
\end{array}\right.
$$

Considering the third and fourth equations of (5), we have

$$
\left\{\begin{array}{l}
y_{1}\left((n+l) \tau^{+}\right)=(1-D) e^{-d_{1} l \tau} y_{1}\left(n \tau^{+}\right)+D e^{-d_{2} l \tau} y_{2}\left(n \tau^{+}\right) \\
y_{2}\left((n+l) \tau^{+}\right)=D e^{-d_{1} l \tau} y_{1}\left(n \tau^{+}\right)+(1-D) e^{-d_{2} l \tau} y_{2}\left(n \tau^{+}\right)
\end{array}\right.
$$

Considering the fifth and sixth equations of (5), we also have

$$
\left\{\begin{array}{l}
y_{1}\left((n+1) \tau^{+}\right)=y_{1}\left((n+l) \tau^{+}\right) e^{-d_{1}(1-l) \tau}+\mu_{1} \\
y_{2}\left((n+1) \tau^{+}\right)=y_{2}\left((n+l) \tau^{+}\right) e^{-d_{2}(1-l) \tau}+\mu_{2}
\end{array}\right.
$$

Substituting (7) into (8), we have the stroboscopic map of (5)

$$
\left\{\begin{array}{l}
y_{1}\left((n+1) \tau^{+}\right)=(1-D) e^{-d_{1} \tau} y_{1}\left(n \tau^{+}\right)+D e^{-\left[d_{1}(1-l)+d_{2} l\right] \tau} y_{2}\left(n \tau^{+}\right)+\mu_{1} \\
y_{2}\left((n+1) \tau^{+}\right)=D e^{-\left[d_{1} l+d_{2}(1-l)\right] \tau} y_{1}\left(n \tau^{+}\right)+(1-D) e^{-d_{2} \tau} y_{2}\left(n \tau^{+}\right)+\mu_{2}
\end{array}\right.
$$

System (9) has one fixed point

$$
\left\{\begin{array}{l}
y_{1}^{*}=\frac{\mu_{2} B_{1}+\mu_{1}\left(1-B_{2}\right)}{\left(1-A_{1}\right)\left(1-B_{2}\right)-A_{2} B_{1}}>0 \\
y_{2}^{*}=\frac{\mu_{1} A_{2}+\mu_{2}\left(1-A_{1}\right)}{\left(1-A_{1}\right)\left(1-B_{2}\right)-A_{2} B_{1}}>0
\end{array}\right.
$$

where

$$
\begin{aligned}
& A_{1}=(1-D) e^{-d_{1} \tau}<1, \\
& B_{1}=D e^{-\left[d_{1}(1-l)+d_{2} l\right] \tau}<1, \\
& A_{2}=D e^{-\left[d_{1} l+d_{2}(1-l)\right] \tau}<1, \\
& B_{2}=(1-D) e^{-d_{2} \tau}<1 .
\end{aligned}
$$


Lemma 3.5 The fixed point $\left(y_{1}^{*}, y_{2}^{*}\right)$ of $(9)$ is globally asymptotically stable.

Proof For convenience, we denote $\left(y_{1}^{n}, y_{2}^{n}\right)=\left(y_{1}\left(n \tau^{+}\right), y_{2}\left(n \tau^{+}\right)\right)$. The linear form of (9) can be written as

$$
\left(\begin{array}{l}
y_{1}^{n+1} \\
y_{2}^{n+1}
\end{array}\right)=M\left(\begin{array}{l}
y_{1}^{n} \\
y_{2}^{n}
\end{array}\right)
$$

Obviously, the near dynamics of $\left(y_{1}^{*}, y_{2}^{*}\right)$ is determined by linear system (11). The stability of $\left(y_{1}^{*}, y_{2}^{*}\right)$ is determined by the eigenvalue of $M$ less than 1 . If $M$ satisfies the Jury criteria [34], then we know that the eigenvalue of $M$ is less than 1 ,

$$
1-\operatorname{tr} M+\operatorname{det} M>0
$$

We easily see that $\left(y_{1}^{*}, y_{2}^{*}\right)$ is a unique fixed point of $(9)$ and

$$
M=\left(\begin{array}{ll}
A_{1} & B_{1} \\
A_{2} & B_{2}
\end{array}\right) .
$$

Since

$$
\begin{aligned}
1- & \operatorname{tr} M+\operatorname{det} M \\
& =1-\left(A_{1}+B_{2}\right)+\left(A_{1} B_{2}-A_{2} B_{1}\right) \\
& =\left(1-A_{1}\right)\left(1-B_{2}\right)-A_{2} B_{1} \\
& =\left[1-(1-D) e^{-d_{1} \tau}\right] \times\left[1-(1-D) e^{-d_{2} \tau}\right]-D^{2} e^{-\left(d_{1}+d_{2}\right) \tau} \\
& =\left[\left(1-e^{-d_{1} \tau}\right)+D e^{-d_{1} \tau}\right]\left[\left(1-e^{-d_{2} \tau}\right)+D e^{-d_{2} \tau}\right]-D^{2} e^{-\left(d_{1}+d_{2}\right) \tau} \\
& =\left(1-e^{-d_{1} \tau}\right) \times\left(1-e^{-d_{2} \tau}\right)+D e^{-d_{2} \tau}\left(1-e^{-d_{1} \tau}\right)+D e^{-d_{1} \tau}\left(1-e^{-d_{2} \tau}\right) \\
& >0
\end{aligned}
$$

by the Jury criteria, $\left(y_{1}^{*}, y_{2}^{*}\right)$ is locally stable, and then, it is globally asymptotically stable. This completes the proof.

Lemma 3.6 The periodic solution $\left(\widetilde{y_{1}(t)}, \widetilde{y_{2}(t)}\right)$ of system (5) is globally asymptotically stable, where

$$
\left\{\begin{array}{l}
\widetilde{y_{1}(t)}= \begin{cases}y_{1}^{*} e^{-d_{1}(t-n \tau)}, & t \in(n \tau,(n+l) \tau], \\
y_{1}^{* *} e^{-d_{1}(t-(n+l) \tau)}, & t \in((n+l) \tau,(n+1) \tau],\end{cases} \\
\widetilde{y_{2}(t)}= \begin{cases}y_{2}^{*} e^{-d_{2}(t-n \tau)}, & t \in(n \tau,(n+l) \tau], \\
y_{2}^{* *} e^{-d_{2}(t-(n+l) \tau)}, & t \in((n+l) \tau,(n+1) \tau],\end{cases}
\end{array}\right.
$$

where $y_{1}^{*}$ and $y_{2}^{*}$ are determined as in (10), and $y_{1}^{* *}$ and $y_{2}^{* *}$ are defined as

$$
\left\{\begin{array}{l}
y_{1}^{* *}=(1-D) e^{-d_{1} l \tau} y_{1}^{*}+D e^{-d_{2} l \tau} y_{2}^{*}, \\
y_{2}^{* *}=D e^{-d_{1} l \tau} y_{1}^{*}+(1-D) e^{-d_{2} l \tau} y_{2}^{*} .
\end{array}\right.
$$




\section{The dynamics}

\section{Theorem 4.1 If}

$$
D<\frac{1}{2}
$$

and

$$
\max _{i=1,2}\left\{a_{i} \tau-\frac{\beta_{i}\left[y_{i}^{*}\left(1-e^{-d_{i} l \tau}\right)+y_{i}^{* *}\left(1-e^{-d_{i}(1-l) \tau}\right)\right]}{\sigma_{i} d_{i}}\right\}<0 \quad(i=1,2)
$$

then the prey-extinction boundary periodic solution $\left(0, \widetilde{y_{1}(t)}, 0, \widetilde{y_{2}(t)}\right)$ of $(3)$ is globally asymptotically stable, where $y_{i}^{*}(i=1,2)$ and $y_{i}^{* *}(i=1,2)$ are defined by $(10)$ and $(15)$.

Proof First, we prove the local stability of the prey-extinction boundary periodic solution $\left(0, \widetilde{y_{1}(t)}, 0, \widetilde{y_{2}(t)}\right)$ of (3). Defining $x_{1}(t)=x_{1}(t), y_{11}(t)=y_{1}(t)-\widetilde{y_{1}(t)}, x_{2}(t)=x_{2}(t), y_{12}(t)=$ $y_{2}(t)-\widetilde{y_{2}(t)}$, we have the following linearly similar system for (3), which has one periodic solution $\left(0, \widetilde{y_{1}(t)}, 0, \widetilde{y_{2}(t)}\right)$ :

$$
\left(\begin{array}{c}
\frac{d x_{1}(t)}{d t} \\
\frac{d y_{11}(t)}{d t} \\
\frac{d x_{2}(t)}{d t} \\
\frac{d y_{12}(t)}{d t}
\end{array}\right)=\left(\begin{array}{cccc}
a_{1}-\frac{\beta_{1} \widetilde{y_{1}(t)}}{\sigma_{1}} & 0 & 0 & 0 \\
\frac{k_{1} \beta_{1} y_{1}(t)}{\sigma_{1}} & -d_{1} & 0 & 0 \\
0 & 0 & a_{2}-\frac{\beta_{2} \widetilde{2_{2}(t)}}{\sigma_{2}} & 0 \\
0 & 0 & \frac{k_{2} \beta_{2} y_{2}(t)}{\sigma_{2}} & -d_{2}
\end{array}\right)\left(\begin{array}{c}
x_{1}(t) \\
y_{11}(t) \\
x_{2}(t) \\
y_{12}(t)
\end{array}\right)
$$

It is easy to obtain the fundamental matrix

$$
\Phi(t)=\left(\begin{array}{cccc}
\exp \left[\int_{0}^{t}\left(a_{1}-\frac{\beta_{1} y_{1}(s)}{\sigma_{1}}\right) d s\right] & 0 & 0 & 0 \\
\exp \left[\int_{0}^{t} \frac{k_{1} \beta_{1} y_{1}(s)}{\sigma_{1}} d s\right] & \exp \left(-d_{1} t\right) & 0 & 0 \\
0 & 0 & \exp \left[\int_{0}^{t}\left(a_{2}-\frac{\beta_{2} \widetilde{y_{2}(s)}}{\sigma_{2}}\right) d s\right] & 0 \\
0 & 0 & \exp \left[\int_{0}^{t} \frac{k_{2} \beta_{2} y_{2}(s)}{\sigma_{2}} d s\right] & \exp \left(-d_{2} t\right)
\end{array}\right) .
$$

The linearization of the fifth, sixth, seventh, and eighth equations of (3) is

$$
\left(\begin{array}{l}
x_{1}\left((n+l) \tau^{+}\right) \\
y_{11}\left((n+l) \tau^{+}\right) \\
x_{2}\left((n+l) \tau^{+}\right) \\
y_{12}\left((n+l) \tau^{+}\right)
\end{array}\right)=\left(\begin{array}{cccc}
1 & 0 & 0 & 0 \\
0 & 1-D & 0 & D \\
0 & 0 & 1 & 0 \\
0 & D & 0 & 1-D
\end{array}\right)\left(\begin{array}{l}
x_{1}((n+l) \tau) \\
y_{11}((n+l) \tau) \\
x_{2}((n+l) \tau) \\
y_{12}((n+l) \tau)
\end{array}\right) .
$$

The linearization of the ninth, tenth, eleventh, and twelfth equations of (3) is

$$
\left(\begin{array}{l}
x_{1}\left((n+1) \tau^{+}\right) \\
y_{11}\left((n+1) \tau^{+}\right) \\
x_{2}\left((n+1) \tau^{+}\right) \\
y_{12}\left((n+1) \tau^{+}\right)
\end{array}\right)=\left(\begin{array}{llll}
1 & 0 & 0 & 0 \\
0 & 1 & 0 & 0 \\
0 & 0 & 1 & 0 \\
0 & 0 & 0 & 1
\end{array}\right)\left(\begin{array}{l}
x_{1}((n+1) \tau) \\
y_{11}((n+1) \tau) \\
x_{2}((n+1) \tau) \\
y_{12}((n+1) \tau)
\end{array}\right) .
$$


The stability of the periodic solution $\left(0, \widetilde{y_{1}(t)}, 0, \widetilde{y_{2}(t)}\right)$ is determined by the eigenvalues of

$$
M=\left(\begin{array}{cccc}
1 & 0 & 0 & 0 \\
0 & 1-D & 0 & D \\
D & 0 & 1 & 0 \\
0 & D & 0 & 1-D
\end{array}\right)\left(\begin{array}{cccc}
1 & 0 & 0 & 0 \\
0 & 1 & 0 & 0 \\
0 & 0 & 1 & 0 \\
0 & 0 & 0 & 1
\end{array}\right) \Phi(\tau)
$$

which are

$$
\begin{aligned}
\lambda_{1} & =\exp \left[\int_{0}^{\tau}\left(a_{1}-\frac{\beta_{1} \widetilde{y_{1}(s)}}{\sigma_{1}}\right) d s\right], \\
\lambda_{2} & =\left|\frac{(1-D)\left(K_{1}+K_{3}\right)+\sqrt{(1-D)^{2}\left(K_{1}+K_{3}\right)^{2}-4(1-2 D) K_{1} K_{3}}}{2}\right| \\
& \leq\left|\frac{(1-D)\left(K_{1}+K_{3}\right)+\sqrt{(1+D)^{2}\left(K_{1}+K_{3}\right)^{2}}}{2}\right| \\
& \leq\left|\frac{\left(K_{1}+K_{3}\right)}{2}\right|<1, \\
\lambda_{3} & =\exp \left[\int_{0}^{\tau}\left(a_{2}-\frac{\beta_{2} y_{2}(s)}{\sigma_{2}}\right) d s\right],
\end{aligned}
$$

and

$$
\begin{aligned}
\lambda_{4} & =\left|\frac{(1-D)\left(K_{1}+K_{3}\right)-\sqrt{(1-D)^{2}\left(K_{1}+K_{3}\right)^{2}-4(1-2 D) K_{1} K_{3}}}{2}\right| \\
& \leq\left|\frac{(1-D)\left(K_{1}+K_{3}\right)-\sqrt{(1-D)^{2}\left(K_{1}-K_{3}\right)^{2}}}{2}\right| \\
& =\left|\frac{(1-D)\left(K_{1}+K_{3}\right)-(1-D)\left|K_{1}-K_{3}\right|}{2}\right| \\
& \leq(1-D) \max \left\{K_{1}, K_{3}\right\}<1,
\end{aligned}
$$

where $K_{1}=e^{-d_{1} \tau}<1, K_{3}=e^{-d_{2} \tau}<1$, and condition (16) holds. According to conditions (16), (17), and the Floquet theory [21], if

$$
\exp \left[\int_{0}^{\tau}\left(a_{i}-\frac{\beta_{i} \widetilde{y_{i}(s)}}{\sigma_{i}}\right) d s\right]<1 \quad(i=1,2)
$$

then

$$
\lambda_{1}<1
$$

and

$$
\lambda_{3}<1,
$$

and thus the prey-extinction boundary periodic solution $\left(0, \widetilde{y_{1}(t)}, 0, \widetilde{y_{2}(t)}\right)$ of $(3)$ is locally stable. 
In the following, we will prove the global attraction. By condition (17) we can choose $\varepsilon>0$ such that

$$
\rho_{i}=\exp \left[\int_{0}^{\tau}\left(a_{i}-\frac{\beta_{i}\left(\widetilde{y_{i}(s)}-\varepsilon\right)}{\sigma_{i}}\right) d s\right]<1 \quad(i=1,2) .
$$

From the second and fourth equations of (3) we notice that $\frac{d y_{i}(t)}{d t} \geq-d_{i} y_{i}(t)(i=1,2)$. Then, we consider following impulsive comparative differential equation:

$$
\left\{\begin{array}{l}
\frac{d y_{21}(t)}{d t}=-d_{1} y_{21}(t), \\
\left.\begin{array}{l}
\frac{d y_{22}(t)}{d t}=-d_{2} y_{22}(t), \\
\Delta y_{21}(t)=D\left(y_{22}(t)-y_{21}(t)\right), \\
\Delta y_{22}(t)=D\left(y_{21}(t)-y_{22}(t)\right),
\end{array}\right\} \quad t=(n+l) \tau, t \neq(n+1) \tau, \\
\Delta y_{21}(t)=\mu_{1}, \\
\Delta y_{22}(t)=\mu_{2},
\end{array}\right\} \quad t=(n+1) \tau .
$$

From Lemma 3.6 and the comparison theorem of impulsive equation (see Theorem 3.1.1 in [21]) we have $y_{1}(t) \geq y_{21}(t), y_{2}(t) \geq y_{22}(t)$, and $y_{21}(t) \rightarrow \widetilde{y_{1}(t)}, y_{22}(t) \rightarrow \widetilde{y_{2}(t)}$ as $t \rightarrow \infty$. Then

$$
\left\{\begin{array}{l}
y_{1}(t) \geq y_{21}(t) \geq \widetilde{y_{1}(t)}-\varepsilon \\
y_{2}(t) \geq y_{22}(t) \geq \widetilde{y_{2}(t)}-\varepsilon
\end{array}\right.
$$

for $t$ large enough. For convenience, we may assume that (19) holds for all $t \geq 0$. From (3) and (19) we get

$$
\frac{d x_{i}(t)}{d t} \leq\left[a_{i}-\frac{\beta_{i}\left(\widetilde{y_{i}(t)}-\varepsilon\right)}{\sigma_{i}}\right] x_{i}(t) \quad(i=1,2)
$$

So $x_{i}((n+1) \tau) \leq x_{i}\left(n \tau^{+}\right) \exp \left[\int_{n \tau}^{(n+1) \tau}\left(a_{i}-\frac{\beta_{i}\left(\widetilde{\left.y_{i}(s)-\varepsilon\right)}\right.}{\sigma_{i}}\right) d s\right](i=1,2)$. Hence, $x_{i}(n \tau) \leq x_{i}\left(0^{+}\right) \rho_{i}^{n}$ $(i=1,2)$ and $x_{i}(n \tau) \rightarrow 0(i=1,2)$ as $n \rightarrow \infty$; therefore, $x_{i}(t) \rightarrow 0(i=1,2)$ as $t \rightarrow \infty$.

Next, we will prove that $y_{i}(t) \rightarrow \widetilde{y_{i}(t)}(i=1,2)$ as $t \rightarrow \infty$. For $\varepsilon_{1}>0$, there must exist $t_{0}>0$ such that $0<x_{i}(t)<\varepsilon_{1}(i=1,2)$ for all $t \geq t_{0}$. Without loss of generality, we may assume that $0<x_{i}(t)<\varepsilon_{1}$ for all $t \geq 0$. For system (3), we have

$$
-d_{i} y_{i}(t) \leq \frac{d y_{i}(t)}{d t} \leq-\left(d_{i}-\frac{k_{i} \beta_{i} \varepsilon_{1}}{\sigma_{i}+\varepsilon_{1}}\right) y_{i}(t) \quad(i=1,2)
$$

and then we have $y_{21}(t) \leq y_{1}(t) \leq y_{31}(t), y_{22}(t) \leq y_{2}(t) \leq y_{32}(t)$, and $y_{21}(t) \rightarrow \widetilde{y_{1}(t)}, y_{22}(t) \rightarrow$ $\widetilde{y_{2}(t)}, y_{31}(t) \rightarrow \widetilde{y_{31}(t)}, y_{32}(t) \rightarrow \widetilde{y_{32}(t)}$ as $t \rightarrow \infty$, where $\left(y_{21}(t), y_{22}(t)\right)$ and $\left(y_{31}(t), y_{32}(t)\right)$ are the solutions of (18) and

$$
\left\{\begin{array}{l}
\frac{d y_{31}(t)}{d t}=-\left(d_{1}-\frac{k_{1} \beta_{1} \varepsilon_{1}}{\sigma_{1}+\varepsilon_{1}}\right) y_{31}(t), \\
\frac{d y_{32}(t)}{d t}=-\left(d_{2}-\frac{k_{2} \beta_{2} \varepsilon_{1}}{\sigma_{2}+\varepsilon_{1}}\right) y_{32}(t),
\end{array}\right\} \quad t \neq(n+l) \tau, t \neq(n+1) \tau
$$


respectively,

$$
\left\{\begin{array}{l}
\widetilde{y_{31}(t)}=\left\{\begin{array}{ll}
y_{31}^{*} e^{-\left(d_{1}-\frac{k_{1} \beta_{1} \varepsilon_{1}}{\sigma_{1}+\varepsilon_{1}}\right)(t-n \tau)}, & t \in(n \tau,(n+l) \tau], \\
y_{31}^{* *} e^{-\left(d_{1}-\frac{k_{1} \beta_{1} \varepsilon_{1}}{\sigma_{1}+\varepsilon_{1}}\right)(t-(n+l) \tau)}, & t \in((n+l) \tau,(n+1) \tau],
\end{array} \widetilde{\widetilde{y_{32}(t)}}= \begin{cases}y_{32}^{*} e^{-\left(d_{2}-\frac{k_{2} \beta_{2} \varepsilon_{1}}{\sigma_{2}+\varepsilon_{1}}\right)(t-n \tau)}, & t \in(n \tau,(n+l) \tau], \\
y_{32}^{* *} e^{-\left(d_{2}-\frac{k_{2} \beta_{2} \varepsilon_{1}}{\sigma_{2}+\varepsilon_{1}}\right)(t-(n+l) \tau)}, & t \in((n+l) \tau,(n+1) \tau],\end{cases} \right.
\end{array}\right.
$$

where $y_{31}^{*}$ and $y_{32}^{*}$ are determined as

$$
\left\{\begin{array}{l}
y_{31}^{*}=\frac{\mu_{2} B_{31}+\mu_{1}\left(1-B_{32}\right)}{\left(1-A_{31}\right)\left(1-B_{32}\right)-A_{32} B_{31}}>0 \\
y_{32}^{*}=\frac{\mu_{1} A_{32}+\mu_{2}\left(1-A_{31}\right)}{\left(1-A_{31}\right)\left(1-B_{32}\right)-A_{32} B_{31}}>0
\end{array}\right.
$$

and $y_{31}^{* *}$ and $y_{32}^{* *}$ are defined as

$$
\left\{\begin{array}{l}
y_{31}^{* *}=(1-D) e^{-\left(d_{1}-\frac{k_{1} \beta_{1} \varepsilon_{1}}{\sigma_{1}+\varepsilon_{1}}\right) l \tau} y_{31}^{*}+D e^{-\left(d_{2}-\frac{k_{2} \beta_{2} \varepsilon_{1}}{\sigma_{2}+\varepsilon_{1}}\right) l \tau} y_{32}^{*}, \\
y_{32}^{* *}=D e^{-\left(d_{1}-\frac{k_{1} \beta_{1} \varepsilon_{1}}{\sigma_{1}+\varepsilon_{1}}\right) l \tau} y_{31}^{*}+(1-D) e^{-\left(d_{2}-\frac{k_{2} \beta_{2} \varepsilon_{1}}{\sigma_{2}+\varepsilon_{1}}\right) l \tau} y_{32}^{*},
\end{array}\right.
$$

where

$$
\begin{aligned}
& A_{31}=(1-D) e^{-\left(d_{1}-\frac{k_{1} \beta_{1} \varepsilon_{1}}{\sigma_{1}+\varepsilon_{1}}\right) \tau}<1, \\
& B_{31}=D e^{-\left[\left(d_{1}-\frac{k_{1} \beta_{1} \varepsilon_{1}}{\sigma_{1}+\varepsilon_{1}}\right)(1-l)+\left(d_{2}-\frac{k_{2} \beta_{2} \varepsilon_{1}}{\sigma_{2}+\varepsilon_{1}}\right)\right] \tau}<1, \\
& A_{32}=D e^{-\left[\left(d_{1}-\frac{k_{1} \beta_{1} \varepsilon_{1}}{\sigma_{1}+\varepsilon_{1}}\right) l+\left(d_{2}-\frac{k_{2} \beta_{2} \varepsilon_{1}}{\sigma_{2}+\varepsilon_{1}}\right)(1-l)\right] \tau}<1, \\
& B_{32}=(1-D) e^{-\left(d_{2}-\frac{k_{2} \beta_{2} \varepsilon_{1}}{\sigma_{2}+\varepsilon_{1}}\right) \tau}<1 .
\end{aligned}
$$

For any $\varepsilon_{2}>0$, there exists $t_{1}, t>t_{1}$, such that

$$
\widetilde{y_{21}(t)}-\varepsilon_{2}<y_{1}(t)<\widetilde{y_{31}(t)}+\varepsilon_{2}
$$

and

$$
\widetilde{y_{22}(t)}-\varepsilon_{2}<y_{2}(t)<\widetilde{y_{32}(t)}+\varepsilon_{2} \text {. }
$$

Letting $\varepsilon_{1} \rightarrow 0$, we have

$$
\widetilde{y_{1}(t)}-\varepsilon_{2}<y_{1}(t)<\widetilde{y_{1}(t)}+\varepsilon_{2}
$$

and

$$
\widetilde{y_{2}(t)}-\varepsilon_{2}<y_{2}(t)<\widetilde{y_{2}(t)}+\varepsilon_{2}
$$

for $t$ large enough, which implies $y_{1}(t) \rightarrow \widetilde{y_{1}(t)}$ and $y_{2}(t) \rightarrow \widetilde{y_{2}(t)}$ as $t \rightarrow \infty$. This completes the proof.

The next work is to investigate the permanence of system (3). 
Definition 4.2 System (3) is said to be permanent if there are constants $m, M>0$ (independent of initial value) and a finite time $T_{0}$ such that for all solutions $\left(x_{1}(t), y_{1}(t), x_{2}(t)\right.$, $\left.y_{2}(t)\right)$ with any initial values $x_{1}\left(0^{+}\right)>0, y_{1}\left(0^{+}\right)>0, x_{2}\left(0^{+}\right)>0, y_{2}\left(0^{+}\right)>0$, we have $m \leq$ $x_{1}(t) \leq M, m \leq y_{1}(t) \leq M, m \leq x_{2}(t) \leq M, m \leq y_{2}(t) \leq M$ for all $t \geq T_{0}$. Here $T_{0}$ may depend on the initial values $\left(x_{1}\left(0^{+}\right), y_{1}\left(0^{+}\right), x_{2}\left(0^{+}\right), y_{2}\left(0^{+}\right)\right)$.

Theorem 4.3 If

$$
\min _{i=1,2}\left\{a_{i} \tau-\frac{\beta_{i}\left[y_{i}^{*}\left(1-e^{-d_{i} l \tau}\right)+y_{i}^{* *}\left(1-e^{-d_{i}(1-l) \tau}\right)\right]}{\sigma_{i} d_{i}}\right\}>0 \quad(i=1,2),
$$

then system (3) is permanent, where $y_{i}^{*}(i=1,2)$ and $y_{i}^{* *}(i=1,2)$ are defined by (10) and (15), respectively.

Proof Suppose $\left(x_{1}(t), y_{1}(t), x_{2}(t), y_{2}(t)\right)$ is a solution of $(3)$ with $x_{1}(0)>0, y_{1}(0)>0, x_{2}(0)>0$, $y_{2}(0)>0$. By Lemma 3.4 there exists a constant $M>0$ such that $x_{1}(t) \leq M, y_{1}(t) \leq M$, $x_{2}(t) \leq M, y_{2}(t) \leq M$ for $t$ large enough. From (3) and Theorem 4.1 we have $y_{i}(t)>$ $\widetilde{y_{i}(t)}-\varepsilon_{2}>y_{i}^{*} e^{-d_{i} l \tau}+y_{i}^{* *} e^{-d_{i}(1-l) \tau} \triangleq m_{i}(i=1,2)$ for $\varepsilon_{2}$ small enough. So we only need to find $m_{3}>0$ and $\varepsilon_{3}$ such that $x_{i}(t)>m_{3}$ for $t$ large enough. Otherwise, we can select $m_{4}>0$ small enough satisfying $m_{4}<\frac{\sigma_{i} d_{i}}{k_{i} \beta_{i}-d_{i}}\left(d_{i}<k_{i} \beta_{i}\right)$ and prove that $x_{i}(t)<m_{4}$ cannot hold for $t \geq 0$. Suppose the contrary. By condition (26), choosing $\varepsilon_{3}$ small enough, we can obtain

$$
\delta_{i}=a_{i} \tau-\frac{\beta_{i}\left[y_{4 i}^{*}\left(1-e^{-\left(d_{i}-\frac{k_{i} \beta_{i} m_{4}}{\sigma_{i}+m_{4}}\right) l \tau}\right)+y_{4 i}^{* *}\left(1-e^{-\left(d_{i}-\frac{k_{i} \beta_{i} m_{4}}{\sigma_{i}+m_{4}}\right)(1-l) \tau}\right)\right]}{\sigma_{i}\left(d_{i}-\frac{k_{i} \beta_{i} m_{4}}{\sigma_{i}+m_{4}}\right)}-\frac{\beta_{i} \varepsilon_{3}}{\sigma_{i}} \tau>0
$$

with $y_{4 i}^{*}(i=1,2)$ and $y_{4 i}^{* *}(i=1,2)$ are defined by (30) and (31). Then,

$$
\left\{\begin{array}{l}
\frac{d y_{1}(t)}{d t}<-\left(d_{1}-\frac{k_{1} \beta_{1} m_{4}}{\sigma_{1}+m_{4}}\right) y_{1}(t), \\
\frac{d y_{2}(t)}{d t}<-\left(d_{2}-\frac{k_{2} \beta_{2} m_{4}}{\sigma_{2}+m_{4}}\right) y_{2}(t), \\
\Delta y_{1}(t)=D\left(y_{2}(t)-y_{1}(t)\right), \\
\Delta y_{2}(t)=D\left(y_{1}(t)-y_{2}(t)\right), \\
\Delta y_{1}(t)=\mu_{1}, \\
\Delta y_{2}(t)=\mu_{2},
\end{array}\right\} \quad t=(n+1) \tau .
$$

By Lemma 3.6 we have $y_{1}(t) \leq y_{41}(t), y_{2}(t) \leq y_{42}(t)$ and $y_{41}(t) \rightarrow \overline{y_{41}(t)}, y_{42}(t) \rightarrow \overline{y_{42}(t)}$, $t \rightarrow \infty$, where $\left(y_{41}(t), y_{42}(t)\right)$ is the solution of

$$
\left\{\begin{array}{l}
\frac{d y_{41}(t)}{d t}=-\left(d_{1}-\frac{k_{1} \beta_{1} m_{4}}{\sigma_{1}+m_{4}}\right) y_{41}(t), \\
\frac{d y_{42}(t)}{d t}=-\left(d_{2}-\frac{k_{2} \beta_{2} m_{4}}{\sigma_{2}+m_{4}}\right) y_{42}(t),
\end{array}\right\} \quad t \neq(n+l) \tau, t \neq(n+1) \tau,
$$


with

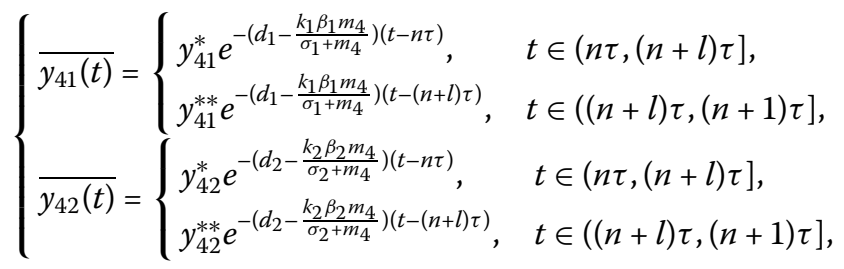

where $y_{41}^{*}$ and $y_{42}^{*}$ are determined as

$$
\left\{\begin{array}{l}
y_{41}^{*}=\frac{\mu_{2} B_{41}+\mu_{1}\left(1-B_{42}\right)}{\left(1-A_{41}\right)\left(1-B_{42}\right)-A_{42} B_{41}}>0 \\
y_{42}^{*}=\frac{\mu_{1} A_{42}+\mu_{2}\left(1-A_{41}\right)}{\left(1-A_{41}\right)\left(1-B_{42}\right)-A_{42} B_{41}}>0
\end{array}\right.
$$

and $y_{41}^{* *}, y_{42}^{* *}$ are defined as

$$
\left\{\begin{array}{l}
y_{41}^{* *}=(1-D) e^{-\left(d_{1}-\frac{k_{1} \beta_{1} m_{4}}{\sigma_{1}+m_{4}}\right) l \tau} y_{41}^{*}+D e^{-\left(d_{2}-\frac{k_{2} \beta_{2} m_{4}}{\sigma_{2}+m_{4}}\right) l \tau} y_{42}^{*}, \\
y_{42}^{* *}=D e^{-\left(d_{1}-\frac{k_{1} \beta_{1} m_{4}}{\sigma_{1}+m_{4}}\right) l \tau} y_{41}^{*}+(1-D) e^{-\left(d_{2}-\frac{k_{2} \beta_{2} m_{4}}{\sigma_{2}+m_{4}}\right) l \tau} y_{42}^{*},
\end{array}\right.
$$

where

$$
\begin{aligned}
& A_{41}=(1-D) e^{-\left(d_{1}-\frac{k_{1} \beta_{1} m_{4}}{\sigma_{1}+m_{4}}\right) \tau}<1, \\
& B_{41}=D e^{-\left[\left(d_{1}-\frac{k_{1} \beta_{1} m_{4}}{\sigma_{1}+m_{4}}\right)(1-l)+\left(d_{2}-\frac{k_{2} \beta_{2} m_{4}}{\sigma_{2}+m_{4}}\right) l\right] \tau}<1, \\
& A_{42}=D e^{-\left[\left(d_{1}-\frac{k_{1} \beta_{1} m_{4}}{\sigma_{1}+m_{4}}\right) l+\left(d_{2}-\frac{k_{2} \beta_{2} m_{4}}{\sigma_{2}+m_{4}}\right)(1-l)\right] \tau}<1, \\
& B_{42}=(1-D) e^{-\left(d_{2}-\frac{k_{2} \beta_{2} m_{4}}{\sigma_{2}+m_{4}}\right) \tau}<1 .
\end{aligned}
$$

Therefore, there exist $T_{1}>0$ and $\varepsilon_{3}>0$ such that

$$
y_{1}(t) \leq y_{41}(t) \leq \overline{y_{41}(t)}+\varepsilon_{3}
$$

and

$$
y_{2}(t) \leq y_{42}(t) \leq \overline{y_{42}(t)}+\varepsilon_{3} .
$$

Then,

$$
\frac{d x_{i}(t)}{d t} \geq\left[a_{i}-\frac{\beta_{i}\left(\overline{y_{4 i}(t)}+\varepsilon_{3}\right)}{\sigma_{i}}\right] x_{i}(t) \quad(i=1,2)
$$

for $t \geq T_{1}$. Let $N_{1} \in N$ and $N_{1} \tau>T_{1}$. Integrating (32) on $(n \tau,(n+1) \tau), n \geq N_{1}$, we have

$$
\begin{aligned}
x_{i}((n+1) \tau) & \geq x_{i}\left(n \tau^{+}\right) \exp \left(\int_{n \tau}^{(n+1) \tau}\left[a_{i}-\frac{\beta_{i}\left(\overline{y_{4 i}(t)}+\varepsilon_{3}\right)}{\sigma_{i}}\right] d t\right) \\
& =x_{i}(n \tau) e^{\delta_{i}} \quad(i=1,2) .
\end{aligned}
$$


Then, $x_{i}\left(\left(N_{1}+k\right) \tau\right) \geq x_{i}\left(N_{1} \tau^{+}\right) e^{k \delta_{i}} \rightarrow \infty$ as $k \rightarrow \infty$, which is a contradiction to the boundedness of $x_{i}(t)(i=1,2)$. Hence, there exists $t_{1}>0$ such that $x_{i}(t) \geq m_{3}(i=1,2)$. This completes the proof.

\section{Simulation analysis and discussion}

In this paper, we establish a predator-prey model with impulsive diffusion and releasing on predator population. This predator-prey model for two regions, which are connected by diffusion of predator population, portrays the evolvement of population. We prove that all solutions of the investigated system are uniformly ultimately bounded. By Theorem 4.1, if $D<\frac{1}{2}$ and

$$
\max _{i=1,2}\left\{a_{i} \tau-\frac{\beta_{i}\left[y_{i}^{*}\left(1-e^{-d_{i} l \tau}\right)+y_{i}^{* *}\left(1-e^{-d_{i}(1-l) \tau}\right)\right]}{\sigma_{i} d_{i}}\right\}<0 \quad(i=1,2)
$$

then the prey-extinction boundary periodic solution $\left(\widetilde{0, y_{1}(t)}, 0, \widetilde{y_{2}(t)}\right)$ of system $(3)$ is globally asymptotically stable. By Theorem 4.3 , if

$$
\min _{i=1,2}\left\{a_{i} \tau-\frac{\beta_{i}\left[y_{i}^{*}\left(1-e^{-d_{i} l \tau}\right)+y_{i}^{* *}\left(1-e^{-d_{i}(1-l) \tau}\right)\right]}{\sigma_{i} d_{i}}\right\}>0 \quad(i=1,2),
$$

then system (3) is permanent.

(a)

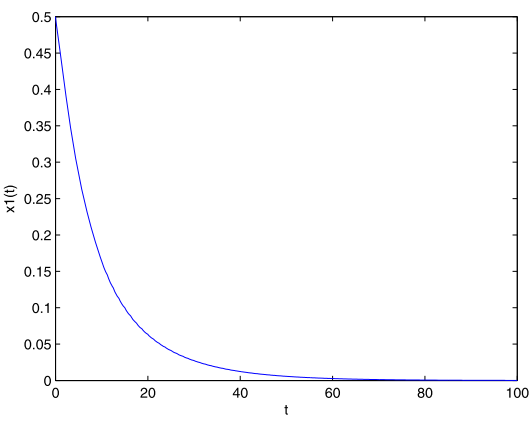

(c)

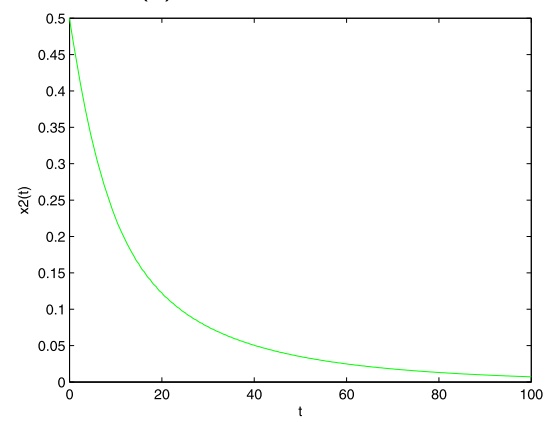

(b)

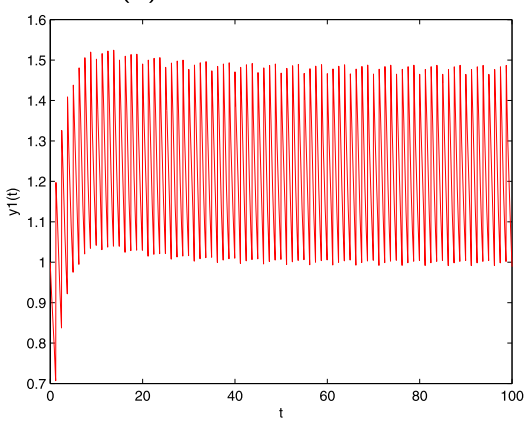

(d)

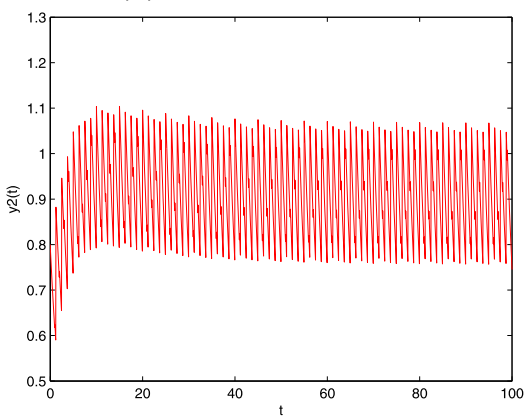

Figure 1 Globally asymptotically stable prey-extinction periodic solution of system (3) with $x_{1}(0)=0.5, y_{1}(0)=0.5, x_{2}(0)=0.5, y_{2}(0)=0.5, a_{1}=0.1, b_{1}=0.2, a_{2}=0.1, b_{2}=0.2, \beta_{1}=0.5, \beta_{2}=5$, $k_{1}=0.5, k_{2}=5, \mu_{1}=0.5, \mu_{2}=0.3, d_{1}=0.3, d_{2}=0.3, \sigma_{1}=3.5, \sigma_{2}=3.5, \tau=1, I=0.25, D=0.1$. (a) Time-series of $x_{1}(t)$; (b) Time-series of $y_{1}(t)$; (c) Time-series of $x_{2}(t)$; (d) Time-series of $y_{2}(t)$. 
(a)

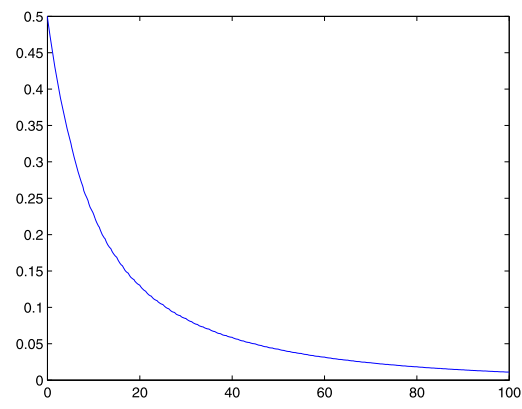

(c)

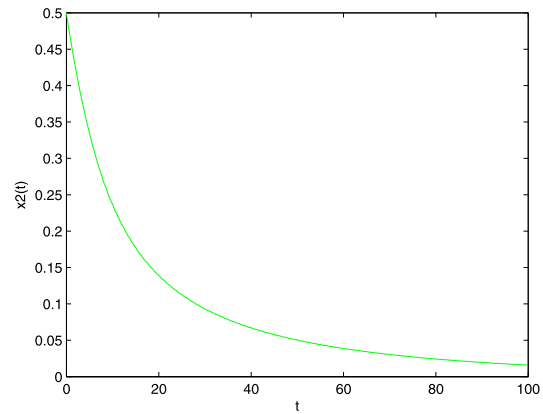

(b)

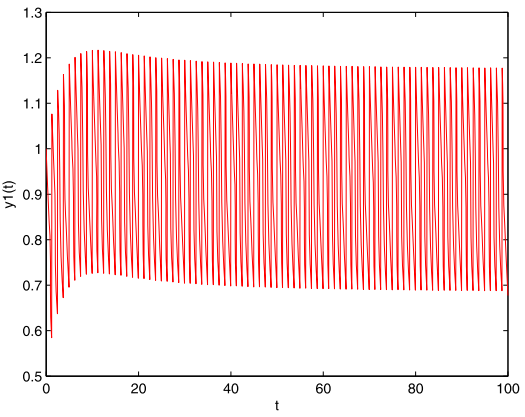

(d)

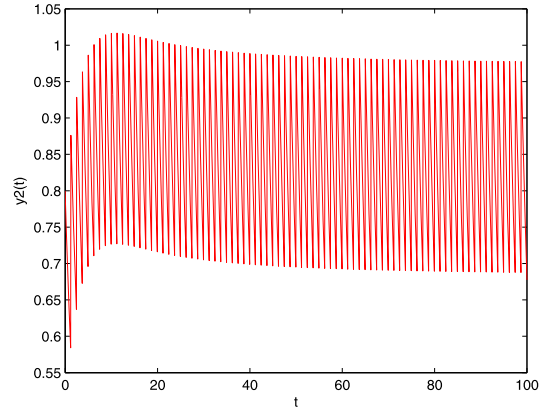

Figure 2 The permanence for system (3) with $x_{1}(0)=0.5, y_{1}(0)=0.5, x_{2}(0)=0.5, y_{2}(0)=0.5, a_{1}=0.1$, $b_{1}=0.2, a_{2}=0.1, b_{2}=0.2, \beta_{1}=0.5, \beta_{2}=5, k_{1}=0.5, k_{2}=5, \mu_{1}=0.5, \mu_{2}=0.3, d_{1}=0.3, d_{2}=0.3$, $\sigma_{1}=3.5, \sigma_{2}=3.5, \tau=1, I=0.25, D=0.95$. (a) Time-series of $x_{1}(t)$; (b) Time-series of $y_{1}(t)$; (c) Time-series of $x_{2}(t) ;(\mathbf{d})$ Time-series of $y_{2}(t)$.

\subsection{The dynamical behaviors influenced by parameter $D$}

Let $x_{1}(0)=0.5, y_{1}(0)=0.5, x_{2}(0)=0.5, y_{2}(0)=0.5, a_{1}=0.1, b_{1}=0.2, a_{2}=0.1, b_{2}=0.2, \beta_{1}=$ $0.5, \beta_{2}=5, k_{1}=0.5, k_{2}=5, \mu_{1}=0.5, \mu_{2}=0.3, d_{1}=0.3, d_{2}=0.3, \sigma_{1}=3.5, \sigma_{2}=3.5, \tau=1$, $l=0.25, D=0.1$. Then conditions (16) and (17) are obviously satisfied, and thus the preyextinction periodic solution of system (3) is globally asymptotically stable (see Figure 1). Also assume that $x_{1}(0)=0.5, y_{1}(0)=0.5, x_{2}(0)=0.5, y_{2}(0)=0.5, a_{1}=0.1, b_{1}=0.2, a_{2}=0.1$, $b_{2}=0.2, \beta_{1}=0.5, \beta_{2}=5, k_{1}=0.5, k_{2}=5, \mu_{1}=0.5, \mu_{2}=0.3, d_{1}=0.3, d_{2}=0.3, \sigma_{1}=3.5$, $\sigma_{2}=3.5, \tau=1, l=0.25, D=0.95$. Then condition (26) is obviously satisfied, and system (3) is permanent (see Figure 2). From (17) and (26) we can calculate that there exists one threshold $D^{*}$, which satisfies

$$
\max _{i=1,2}\left\{a_{i} \tau-\frac{\beta_{i}\left[y_{i}^{*}\left(1-e^{-d_{i} l \tau}\right)+y_{i}^{* *}\left(1-e^{-d_{i}(1-l) \tau}\right)\right]}{\sigma_{i} d_{i}}\right\}<0 \quad(i=1,2)
$$

or

$$
\min _{i=1,2}\left\{a_{i} \tau-\frac{\beta_{i}\left[y_{i}^{*}\left(1-e^{-d_{i} l \tau}\right)+y_{i}^{* *}\left(1-e^{-d_{i}(1-l) \tau}\right)\right]}{\sigma_{i} d_{i}}\right\}>0 \quad(i=1,2) .
$$

If $D>D^{*}$, then the prey population will go to extinction; if $D<D^{*}$, then the population will be permanent. 
(a)

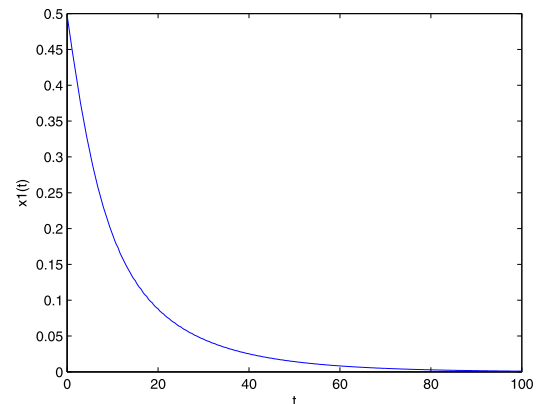

(c)

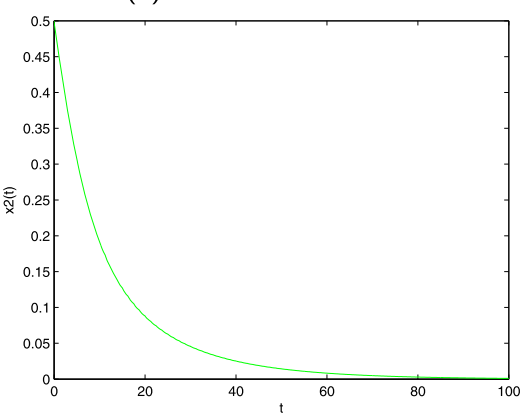

(b)

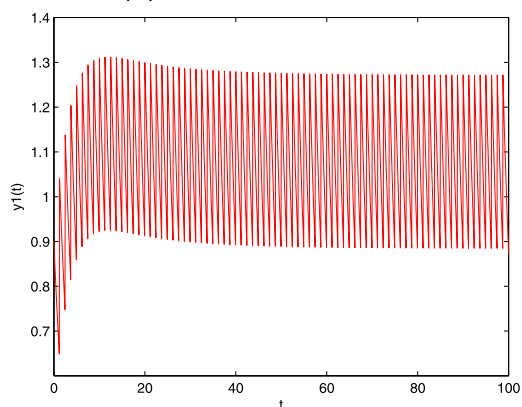

(d)

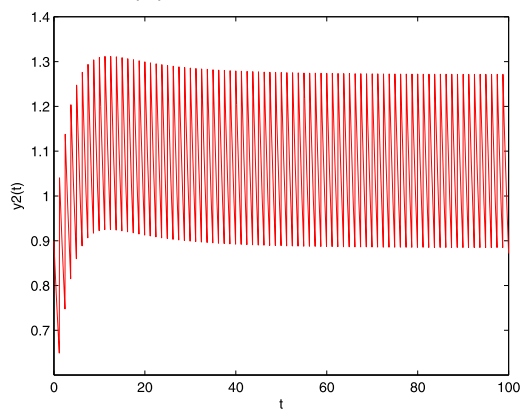

Figure 3 Globally asymptotically stable prey-extinction periodic solution of system (3) with $x_{1}(0)=0.5, y_{1}(0)=0.5, x_{2}(0)=0.5, y_{2}(0)=0.5, a_{1}=0.1, b_{1}=0.2, a_{2}=0.1, b_{2}=0.2, \beta_{1}=0.5, \beta_{2}=5$, $k_{1}=0.5, k_{2}=5, \mu_{1}=0.4, \mu_{2}=0.4, d_{1}=0.4, d_{2}=0.3, \sigma_{1}=3.5, \sigma_{2}=3.5, \tau=1, I=0.25, D=0.2$. (a) Time-series of $x_{1}(t)$; (b) Time-series of $y_{1}(t)$; (c) Time-series of $x_{2}(t)$; (d) Time-series of $y_{2}(t)$.

(a)

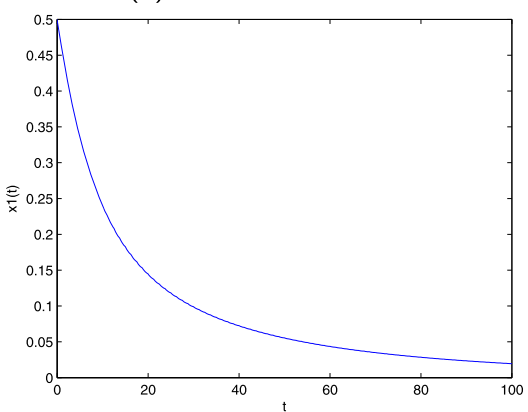

(c)

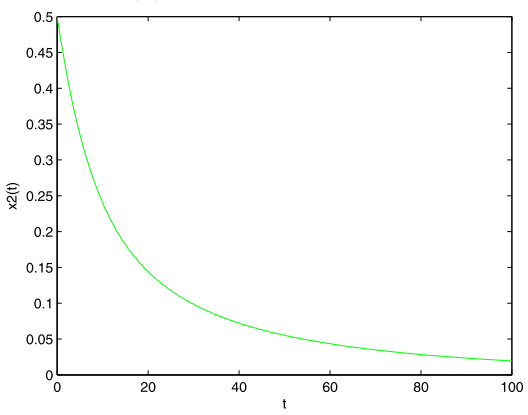

(b)

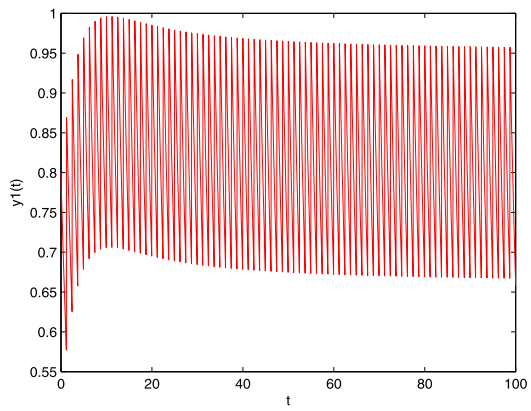

(d)

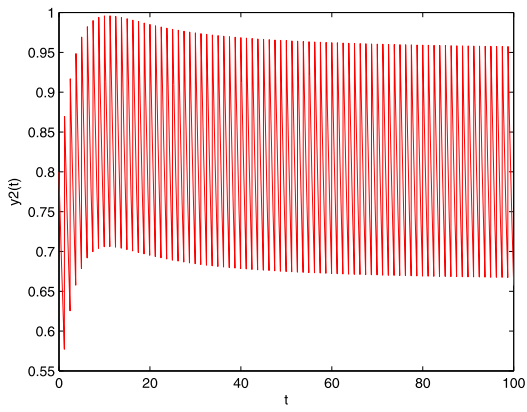

Figure 4 The permanence for system (3) with $x_{1}(0)=0.5, y_{1}(0)=0.5, x_{2}(0)=0.5, y_{2}(0)=0.5, a_{1}=0.1$, $b_{1}=0.2, a_{2}=0.1, b_{2}=0.2, \beta_{1}=0.5, \beta_{2}=5, k_{1}=0.5, k_{2}=5, \mu_{1}=0.3, \mu_{2}=0.3, d_{1}=0.3, d_{2}=0.3$, $\sigma_{1}=3.5, \sigma_{2}=3.5, \tau=1, I=0.25, D=0.2$. (a) Time-series of $x_{1}(t)$; (b) Time-series of $y_{1}(t)$; (c) Time-series of $x_{2}(t) ;(\mathbf{d})$ Time-series of $y_{2}(t)$. 


\subsection{The dynamical behaviors influenced by parameters $\mu_{1}$ and $\mu_{2}$}

In this subsection, we always assume that $\mu=\mu_{1}=\mu_{2}$. Assume that $x_{1}(0)=0.5, y_{1}(0)=0.5$, $x_{2}(0)=0.5, y_{2}(0)=0.5, a_{1}=0.1, b_{1}=0.2, a_{2}=0.1, b_{2}=0.2, \beta_{1}=0.5, \beta_{2}=5, k_{1}=0.5, k_{2}=5$, $\mu_{1}=0.4, \mu_{2}=0.4, d_{1}=0.4, d_{2}=0.3, \sigma_{1}=3.5, \sigma_{2}=3.5, \tau=1, l=0.25, D=0.2$. Then conditions (16) and (17) are obviously satisfied, and the prey-extinction periodic solution of system (3) is globally asymptotically stable (see Figure 3 ). Also, assume that $x_{1}(0)=0.5$, $y_{1}(0)=0.5, x_{2}(0)=0.5, y_{2}(0)=0.5, a_{1}=0.1, b_{1}=0.2, a_{2}=0.1, b_{2}=0.2, \beta_{1}=0.5, \beta_{2}=5, k_{1}=$ $0.5, k_{2}=5, \mu_{1}=0.3, \mu_{2}=0.3, d_{1}=0.3, d_{2}=0.3, \sigma_{1}=3.5, \sigma_{2}=3.5, \tau=1, l=0.25, D=0.2$. Then condition (26) is obviously satisfied, and system (3) is permanent (see Figure 4). We can calculate that there exists at least one threshold $\mu^{*}$ such that if $\mu>\mu^{*}$, then the prey population will go to extinction, and if $\mu<\mu^{*}$, then the population will be permanent.

From the simulations we discover that the increasing diffusive rate of predator population will count against the pest management. We conclude that the impulsive diffusion and releasing predator provide reliable tactic basis for pest management.

\section{Competing interests}

The authors declare that they have no competing interests.

\section{Authors' contributions}

AZ carried out the main part of this article, PS corrected the manuscript, JJ brought forward many suggestions on this article. All authors have read and approved the final manuscript.

\section{Author details}

${ }^{1}$ Institute of Science, School of Mathematics, Suranaree University of Technology, Nakhon, Ratchasima, 30000, Thailand. ${ }^{2}$ Guizhou Key Laboratory of Economic System Simulation, School of Mathematics and Statistics, Guizhou University of Finance and Economics, Guiyang, 550004, P.R. China.

\section{Acknowledgements}

This work was supported by National Natural Science Foundation of China $(11361014,10961008)$.

Received: 19 January 2016 Accepted: 6 April 2016 Published online: 20 April 2016

\section{References}

1. Caltagirone, LE, Doutt, RL: The history of the vedalia beetle importation to California and its impact on the development of biological control. Annu. Rev. Entomol. 34, 1-16 (1989)

2. DeBach, P: Biological Control of Insect Pests and Weeds. Reinhold, New York (1964)

3. DeBach, P, Rosen, D: Biological Control by Natural Enemies, 2nd edn. Cambridge University Press, Cambridge (1991)

4. Barclay, HJ: Models for pest control using predator release, habitat management and pesticide release in combination. J. Appl. Ecol. 19, 337-348 (1982)

5. Murray, JD: Mathematical Biology. Springer, Berlin (1989)

6. Freedman, HJ: Graphical stability, enrichment, and pest control by a natural enemy. Math. Biosci. 31, $207-225$ (1976)

7. Grasman, J, van Herwaarden, OA, Hemerik, L, van Lenteren, JC: A two-component model of host-parasitoid interactions: determination of the size of inundative releases of parasitoids in biological pest control. Math. Biosci. $169,207-216(2001)$

8. Liu, X, Chen, L: Complex dynamics of Holling type II Lotka-Volterra predator-prey system with impulsive perturbations on the predator. Chaos Solitons Fractals 16, 311-320 (2003)

9. Jiao, J, Meng, X, Chen, L: A stage-structured Holling mass defence predator-prey model with impulsive perturbations on predators. Appl. Math. Comput. 189, 1448-1458 (2007)

10. Levin, SA: Dispersion and population interaction. Am. Nat. 108, 207-228 (1994)

11. Allen, LJS: Persistence and extinction in single-species reaction-diffusion models. Bull. Math. Biol. 45(2), 209-227 (1983)

12. Song, $X$, Chen, L: Uniform persistence and global attractivity for nonautonomous competitive systems with dispersion. J. Syst. Sci. Complex. 15, 307-314 (2002)

13. Cui, J, Chen, L: Permanence and extinction in logistic and Lotka-Volterra system with diffusion. J. Math. Anal. Appl. 258(2), 512-535 (2001)

14. Beretta, E, Takeuchi, Y: Global asymptotic stability of Lotka-Volterra diffusion models with continuous time delays. SIAM J. Appl. Math. 48, 627-651 (1998)

15. Beretta, E, Takeuchi, Y: Global stability of single species diffusion Volterra models with continuous time delays. Bull. Math. Biol. 49, 431-448 (1987)

16. Freedman, HI, Takeuchi, Y: Global stability and predator dynamics in a model of prey dispersal in a patchy environment. Nonlinear Anal. 13, 993-1002 (1989)

17. Freedman, HI: Single species migration in two habitats: persistence and extinction. Math. Model. 8, $778-780$ (1987) 
18. Freedman, $\mathrm{HI}$, Rai, B, Waltman, P: Mathematical models of population interactions with dispersal II: differential survival in a change of habitat. J. Math. Anal. Appl. 115, 140-154 (1986)

19. Freedman, HI, Takeuchi, Y: Predator survival versus extinction as a function of dispersal in a predator-prey model with patchy environment. Appl. Anal. 31, 247-266 (1989)

20. Hui, J, Chen, L: A single species model with impulsive diffusion. Acta Math. Appl. Sin. 21(1), 43-48 (2005)

21. Bainov, D, Simeonov, P: Impulsive Differential Equations: Periodic Solution and Applications. Longman, New York (1993)

22. Jiao, J, Chen, L, Cai, S, Wang, L: Dynamics of a stage-structured predator-prey model with prey impulsively diffusing between two patches. Nonlinear Anal., Real World Appl. 11, 2748-2756 (2010)

23. Meng, $\mathrm{X}$, Chen, L: Permanence and global stability in an impulsive Lotka-Volterra $\mathrm{N}$-species competitive system with both discrete delays and continuous delays. Int. J. Biomath. 1, 179-196 (2008)

24. Jiao, J, Chen, L, Luo, G: An appropriate pest management SI model with biological and chemical control concern. Appl. Math. Comput. 196, 285-293 (2008)

25. Jiao, J, Chen, L: Global attractivity of a stage-structure variable coefficients predator-prey system with time delay and impulsive perturbations on predators. Int. J. Biomath. 1, 197-208 (2008)

26. Jiao, J, Pang, G, Chen, L, Luo, G: A delayed stage-structured predator-prey model with impulsive stocking on prey and continuous harvesting on predator. Appl. Math. Comput. 195, 316-325 (2008)

27. Jiao, J, Cai, S, Chen, L: Analysis of a stage-structured predator-prey system with birth pulse and impulsive harvesting at different moments. Nonlinear Anal., Real World Appl. 12, 2232-2244 (2011)

28. Lakshmikantham, V, Bainov, D, Simeonov, P: Theory of Impulsive Differential Equations. World Scientific, Singapore (1989)

29. Chen, L, Meng, X, Jiao, J: Biological Dynamics. Science Press, Beijing (2009) (in Chinese)

30. Gakkhar, S, Negi, K: Pulse vaccination in SIRS epidemic model with non-monotonic incidence rate. Chaos Solitons Fractals 35, 626-638 (2008)

31. Zhou, Y, Liu, H: Stability of periodic solutions for an SIS model with pulse vaccination. Math. Comput. Model. 38 299-308 (2003)

32. Stone, L, Shulgin, B, Agur, Z: Theoretical examination of the pulse vaccination policy in the SIR epidemic models. Math. Comput. Model. 31, 207-215 (2000)

33. Gao, S, Chen, L, Nieto, JJ, Torres, A: Analysis of a delayed epidemic model with pulse vaccination and saturation incidence. Vaccine 24, 6037-6045 (2006)

34. Jury, El: Inners and Stability of Dynamics System. Wiley, New York (1974)

\section{Submit your manuscript to a SpringerOpen ${ }^{\circ}$ journal and benefit from:}

- Convenient online submission

- Rigorous peer review

- Immediate publication on acceptance

- Open access: articles freely available online

High visibility within the field

- Retaining the copyright to your article 